\title{
Effects of regenerative radioelectric asymmetric conveyer treatment on human normal and osteoarthritic chondrocytes exposed to IL-I $\beta$. A biochemical and morphological study
}

\author{
This article was published in the following Dove Press journal: \\ Clinical Interventions in Aging \\ 18 March 2013 \\ Number of times this article has been viewed
}

\author{
Giulia Collodel' \\ Antonella Fioravanti ${ }^{2}$ \\ Nicola Antonio Pascarelli ${ }^{2}$ \\ Antonello Lamboglia ${ }^{2}$ \\ Vania Fontani ${ }^{3}$ \\ Margherita Maioli ${ }^{3-5}$ \\ Sara Santaniello $0^{4,5}$ \\ Gianfranco Pigliaru ${ }^{4,5}$ \\ Alessandro Castagna ${ }^{3}$ \\ Elena Moretti' \\ Francesca lacoponi' \\ Salvatore Rinaldi ${ }^{3}$ \\ Carlo Ventura ${ }^{3,5,6}$ \\ 'Department of Biomedical Sciences \\ (Applied Biology), ${ }^{2}$ Department of \\ Clinical Medicine and Immunological \\ Sciences (Rheumatology Unit), \\ University of Siena, Siena, Italy; \\ ${ }^{3}$ Department of Regenerative \\ Medicine, Rinaldi Fontani Institute, \\ Florence, Italy; ${ }^{4}$ Department of \\ Biomedical Sciences, University of \\ Sassari, Sassari, Italy; ${ }^{5}$ Laboratory \\ of Molecular Biology and Stem \\ Cell Engineering, National Institute \\ of Biostructures and Biosystems, \\ Bologna, Italy; ${ }^{6}$ Cardiovascular \\ Department, S Orsola-Malpighi \\ Hospital, University of Bologna, \\ Bologna, Italy
}

Correspondence: Salvatore Rinald Rinaldi Fontani Institute, Viale Belfiore 43, 50 I 44 Florence, Italy

Tel +39055 290307

Fax +39055 290399

Email srinaldi@irf.it
Purpose: Osteoarthritis (OA) is a degenerative disease characterized by a progressive loss of articular cartilage extracellular matrix and is due to functional impairments occurring in chondrocytes. In previous works, we highlighted that Regenerative Tissue Optimization (TORGN) treatment with radioelectric asymmetric conveyer (REAC) technology influenced the gene expression profiles controlling stem cell differentiation and the pluripotency of human skin-derived fibroblasts in vitro. Since interleukin-1 beta signaling has been implicated in the induction and progression of this disease (through metalloproteinase- 3 synthesis and nitric oxide production), we investigated whether REAC TO-RGN might influence the biochemical and morphological changes induced by interleukin-1 beta in normal and OA chondrocytes.

Methods: The induction of metalloproteinase-3 and proteoglycan synthesis was evaluated by a solid-phase enzyme-amplified sensitivity immunoassay, and nitric oxide production was evaluated with the Griess method. Ultrastructural features were observed by transmission electron microscopy.

Results: REAC TO-RGN treatment decreased nitric oxide and metalloproteinase- 3 production in normal and OA chondrocytes, while inducing an increase in proteoglycan synthesis. OA chondrocytes were more affected by REAC TO-RGN treatment than were normal chondrocytes. Ultrastructural changes confirmed that REAC TO-RGN may counteract the negative effects of interleukin-1 beta incubation.

Conclusion: The results of this in vitro study suggest that REAC TO-RGN treatment may represent a new, promising approach for the management of OA.

Keywords: human chondrocytes ultrastructure, metalloproteinase, nitric oxide, proteoglycans, REAC TO-RGN treatment

\section{Introduction}

Osteoarthritis (OA) is the most common articular disorder of hyaline cartilage and subchondral bone. The disease is responsible for substantial direct and indirect socioeconomic costs. ${ }^{1,2}$ Chondrocytes are responsible for the synthesis and maintenance of extracellular matrix within articular cartilage, as they regulate the equilibrium between synthetic and degradative processes. Loss of homeostasis in favor of catabolic activities leads to the destruction of articular cartilage, and chondrocyte death has been postulated to be a crucial event in the pathogenesis of OA. ${ }^{3,4}$ Interleukin-1 beta (IL-1 $\beta$ ) is a cytokine involved in the processes of cartilage degradation, also acting as a potent inhibitor of extracellular matrix synthesis. In response to IL-1 $\beta$, chondrocytes secrete 
proinflammatory cytokines, chemokines, neutral metalloproteinase (MMP), and nitric oxide (NO). ${ }^{5}$ The latter promotes numerous effects on chondrocytes, including inhibition of proteoglycan (PG) and collagen synthesis, activation of MMPs, as well as induction of apoptosis, and can give rise to free radicals, which are implicated in tissue injury in a variety of degenerative diseases. Furthermore, inhibitors of NO synthesis have been shown to delay the development of clinical and histological signs in various experimental models of OA. ${ }^{6}$

Currently, the optimal management of OA requires a combination of pharmacological and nonpharmacological treatment modalities. ${ }^{7}$ Pharmacologic treatment of OA has been largely confined to analgesics or nonsteroidal antiinflammatory drugs (NSAIDs), which are only symptommodifying agents. These drugs control pain but lack known disease-modifying effects. Furthermore, treatment with NSAIDs is limited by their negative secondary effects on the gastrointestinal tract and on cartilage metabolism. ${ }^{8}$ Identification of a tool capable of counteracting the cartilage degeneration occurring during OA without side effects could represent an interesting approach in OA therapy. In this regard, we have recently observed that Regenerative Tissue Optimization (TO-RGN) treatment with radioelectric asymmetric conveyer (REAC) technology ${ }^{9,10}$ significantly influenced the differentiation capability of mouse embryonic stem cells ${ }^{11}$ as well as the pluripotency and plasticity of human skin-derived fibroblasts. ${ }^{12}$ These findings have suggested the purpose of this study - to clarify whether the positive effect of REAC TO-RGN might be related to a capability to counteract the IL-1 $\beta$-induced signaling pathway, a primary cause of OA. REAC TO-RGN treatment was applied to cultures of normal and OA human articular chondrocytes, in the presence and absence of IL-1 $\beta$. The release of PG, MMP-3, and NO was evaluated, and morphological assessment of chondrocytes, using transmission electron microscopy (TEM), was carried out.

\section{Materials and methods}

\section{Cell culture}

Normal human articular cartilage was obtained from the femoral heads of five subjects with displaced femoral neck fractures (three males and two females), and OA human articular cartilage was obtained from the femoral heads of five patients with OA, defined by the clinical and radiological criteria of the American College of Radiology, ${ }^{13}$ undergoing surgery for total hip prostheses. The mean age of the group was 33 years (range: 20-41) for the normal subjects and 70.6 years (range: 67-75) for the OA patients.
Written consent was signed by each participant in this study, along with the guidelines of the Ethical Committee that had approved the study. These samples were sufficient to provide a large number of cell cultures to perform the study protocol. Normal chondrocytes were obtained from the middle layer of cartilage in the femoral heads, whereas the OA chondrocytes originated from the area adjacent to the OA lesion. OA cartilage was characterized by macroscopic focal fibrillation of the articular surface. Normal cartilage was characterized by a glossy, white, completely smooth surface and a healthy appearance without irregularities. Following surgery, the cartilage was dissected aseptically and minced into small pieces. The fragments were washed in Dulbecco's modified Eagle's medium (DMEM) without phenol red, containing $2 \%$ penicillin/streptomycin solution and $0.2 \%$ amphotericin B (Life Technologies, Carlsbad, CA, USA). The chondrocytes were isolated from the articular cartilage by sequential enzymatic digestion: 30 minutes with $0.1 \%$ hyaluronidase, 1 hour with $0.5 \%$ pronase, and 1 hour with $0.2 \%$ collagenase (Sigma-Aldrich, St Louis, MO, USA), at $37^{\circ} \mathrm{C}$ in the wash solution (DMEM + penicillin/streptomycin solution + amphotericin $\mathrm{B})$. The resulting cell suspension was filtered twice using $70 \mu \mathrm{m}$ nylon mesh, then washed and centrifuged at $700 \mathrm{~g}$ for 10 minutes. As shown by the trypan blue viability test, $90 \%-95 \%$ of the recovered cells were alive. The primary cultures of chondrocytes so obtained were maintained in a humidified atmosphere of $5 \% \mathrm{CO}_{2}$ at $37^{\circ} \mathrm{C}$, for 2 weeks.

\section{REAC technology for therapeutic use}

The REAC is an innovative patented technology ${ }^{9,10}$ for biostimulation and/or bioenhancement that induces weak radioelectric currents in tissue, to induce cell-reprogramming activity. ${ }^{12}$ The model used in this study (BENE Bio Enhancer - Neuro Enhancer ${ }^{\circledR}$; ASMED, Florence, Italy) is specifically designed for regenerative treatments. Recently, using a TO-RGN treatment protocol, the REAC technology was able to drive stem cell pluripotency and differentiation ${ }^{11}$ and cell-reprogramming activity. ${ }^{12}$

\section{REAC TO-RGN treatment protocol}

The REAC device was placed into a $\mathrm{CO}_{2}$ incubator, set with the TO-RGN protocol, ${ }^{11,12}$ and its conveyer electrodes were immersed into separate culture media of human normal and OA chondrocytes, in the presence and absence of IL-1 $\beta$, during 48 hours of culture.

The distance between the emitter (at $2.4 \mathrm{GHz}$ ) and the culture medium was approximately $35 \mathrm{~cm}$. The electromagnetic parameters of a single radiofrequency burst of $200 \mathrm{~ms}$ 
duration were the following: radiated power was $\sim 2 \mathrm{~mW}$, electric field $=0.4 \mathrm{~V} / \mathrm{m}$, magnetic field was $1 \mathrm{~mA} / \mathrm{m}$, specific absorption rate (SAR) was $0.128 \mu \mathrm{W} / \mathrm{g}$; the density of radioelectric current flowing in the culture medium during the REAC single radiofrequency burst was $\mathrm{J}=30 \mu \mathrm{A} / \mathrm{cm}^{2}$.

\section{Exposure of chondrocytes to IL-I $\beta$ and REACTO-RGN}

Normal and OA human articular chondrocytes were cultured in 8-well microplates at a density of $4 \times 10^{4}$ cells/well, with $1 \mathrm{~mL}$ of medium without phenol red, containing $10 \%$ fetal bovine serum (FBS), $200 \mathrm{U} / \mathrm{mL}$ penicillin, $200 \mathrm{mg} / \mathrm{mL}$ streptomycin, $2 \mathrm{mM}$ glutamine (Life Technologies), until they became preconfluent. Chondrocytes were divided into eight groups: normal and OA chondrocytes at basal conditions; normal and OA chondrocytes at basal conditions treated with REAC TO-RGN; normal and OA chondrocytes treated with $5 \mathrm{ng} / \mathrm{mL}$ IL-1 $\beta$ (Sigma-Aldrich); and normal and OA chondrocytes treated with REAC TO-RGN and IL-1 $\beta$. The evaluation of cell viability was carried out in each group at every experimental step, using the Countess Automated Cell Counter (Life Technologies) and trypan blue. After 48 hours of treatment, the media were removed and stored at $-80^{\circ} \mathrm{C}$ for the subsequent detection of PGs and MMP-3. Once terminated the REAC treatment, the cells were fixed for TEM. The supernatants were frozen in part to determine subsequently the MMP-3 and PG. Release of NO was immediately detected before freezing using Griess's assay.

\section{PG assay}

The quantity of PGs was measured in the culture medium by a solid-phase enzyme-amplified sensitivity immunoassay (EASIA) (DIAsource ImmunoAssay SA, Nivelles, Belgium), performed on microtiter plates (Heinegard and Saxne ${ }^{13}$ ). This technique uses two monoclonal antibodies that are directed against keratan sulfate proteoglycans and another monoclonal antibody that is directed against the binding site of hyaluronic acid proteoglycans. Standards or samples containing the PG were reacted with captured monoclonal antibodies (MAbs) coated on the microtiter well and with a monoclonal antibody labeled with horseradish peroxidase (MAb-HRP). After an incubation period allowing the formation of a sandwich (coated MAbs1/PG/MAbs2-HRP), the microtiter plate was washed to remove unbound enzyme-labeled antibodies. Bound enzyme-labeled antibodies were measured through a chromogenic reaction. Then, chromogenic solution (tetramethylbenzidine $+\mathrm{H}_{2} \mathrm{O}_{2}$ ) was added and incubated. The reaction was stopped with the addition of stop solution $(\mathrm{HCl})$, and the microtiter plate was then read at the appropriate wavelength
(450 nm). The amount of substrate turnover was determined colorimetrically by measuring the absorbance, which was proportional to the PG concentration. The assay sensitivity was $0.9 \mathrm{ng} / \mathrm{mL}$. The results obtained for the different culture supernatants were normalized with number the cells. The amount of PG was expressed as $\mathrm{ng} / 10^{6}$ cells.

\section{MMP-3 assay}

MMP-3 was assayed by a solid-phase EASIA (BenderMedsystems, Vienna, Austria). ${ }^{15}$ An anti-human MMP-3 coating antibody was absorbed on microwells; any human MMP-3 present in the sample was then bound to the antibodies adsorbed to the microwells. The substrate turnover was determined colorimetrically by measuring the absorbance, which was proportional to the MMP-3 concentration. The sensitivity of the method was estimated to be $<0.1 \mathrm{ng} / \mathrm{mL}$. The results obtained for the different culture supernatants were normalized to the number of cells. The amount of MMP-3 was expressed as $\mathrm{ng} / 10^{6}$ cells.

\section{Nitrite assay}

The quantity of nitrites in the culture medium was measured by the Griess method ( $1 \%$ sulfanilamide, $0.1 \%$ (napthyl) ethylenediamine dihydrochloride, and 2.5\% phosphoric acid). ${ }^{16}$ Equal volumes $(100 \mu \mathrm{L})$ of supernatant and the Griess reagent were incubated on microplates at room temperature for 15 minutes. The absorbance was measured with a spectrophotometer at $550 \mathrm{~nm}$. The concentration of nitrites was calculated using a standard curve made by successive dilutions with a solution of sodium nitrite in water. The results obtained for the different culture supernatants were normalized to the number of cells. The amount of nitrite was expressed as $\mathrm{ng} / 10^{6}$ cells.

\section{TEM}

Cultures of normal and OA human chondrocytes were fixed in cold Karnovsky fixative and maintained at $4^{\circ} \mathrm{C}$ for 2 hours, postfixed in $1 \%$ buffered osmium tetroxide, then dehydrated in a graded ethanol series, and embedded in epon araldite medium. Ultrathin sections, stained with uranyl acetate and lead citrate, were observed and photographed with a TEM (EM 208; Royal Philips Electronics, Amsterdam, The Netherlands). At least 100 cells from each group were examined.

\section{Morphometric and statistical analysis}

For the morphometric studies, we analyzed sections of three different blocks from each group. For standardization and comparison of the different groups, only medially sectioned chondrocytes were investigated; 100 chondrocytes were 
selected using the nucleus/cytoplasm ratio as the selection criterion. Our analysis was based on an established $\operatorname{method}^{17,18}$ for ultrastructural quantitative evaluation of changes in chondrocyte function. Mitochondria and Golgi bodies were counted and recorded. Moreover, the presence of cytoplasmic vacuolization was reported. This cytoplasmic characteristic is known to be predominant in the presence of negative stimulus, represented by IL-1 $\beta$. To evaluate the presence of vacuolization in medially sectioned chondrocytes, we considered five vacuoles as the point of reference.

Data were expressed as the mean \pm standard deviation (SD) of triplicate values for each experiment. For each group (of normal and OA chondrocyte), the comparisons between the basal condition, REAC TO-RGN alone, IL-1 $\beta$ alone, and REAC TO-RGN + IL-1 $\beta$ treated were analyzed using the Friedman test, followed by Dunn's post hoc test. Normal and OA groups were compared using the Mann-Whitney test, considering the differences, for each group, between the basal condition and REAC TO-RGN, between basal and IL-1 $\beta$, and between basal and REAC TO-RGN + IL-1 $\beta$. A $P$ value $<0.05$ (two-tailed) was considered statistically significant. All the data were analyzed using GraphPad Prism software for Windows (v 5.00; GraphPad Software Inc, La Jolla, CA, USA).

\section{Results}

\section{Viability evaluation}

Analysis of cell viability, detected by trypan blue staining before starting, revealed that more than $95 \%$ of normal chondrocytes and $87 \%$ of OA chondrocytes were viable; after 48 hours, cell viability was $86.67 \%$ in normal and $77.83 \%$ in OA chondrocytes. The treatment with IL- $1 \beta$ significantly affected $(P<0.05)$ chondrocyte viability in both groups of cells (normal, 64.83\%; OA, 61\%). REAC TO-RGN exposure was applied at basal conditions, and cell viability resulted to be increased, particularly in OA chondrocytes (normal, 88.83\%; OA, 86.33\%). When REAC TO-RGN treatment was performed in the presence of IL-1 $\beta$, both normal and OA chondrocytes maintained a remarkable viability (73\% and $72.69 \%$, respectively). It was interesting to note that normal and OA chondrocytes showed a significantly different response to IL- $1 \beta$ and REAC TO-RGN treatment. In particular, OA chondrocytes significantly maintained their viability after REAC TO-RGN treatment, both in the presence or absence of IL-1 $\beta$, as compared with normal chondrocytes in the same experimental conditions $(P<0.01)$.

\section{PGs, MMP-3, and NO evaluation}

The amount of PGs (Figure 1A), MMP-3 (Figure 1B), and NO (Figure 1C) in the culture medium of normal and OA chondrocytes incubated in the different experimental conditions are shown.

The total PG concentration in the culture medium increased in the presence of REAC TO-RGN, both at basal conditions and in IL-1 $\beta$-treated normal and OA chondrocytes (Figure 1A). Noteworthy, OA chondrocytes significantly increased $(P<0.01)$ the levels of PGs in the presence of REAC TO-RGN and IL-1 $\beta$ as compared with normal chondrocytes.

The REAC TO-RGN stimulus that was applied to normal or OA chondrocytes reduced MMP-3 and NO levels,
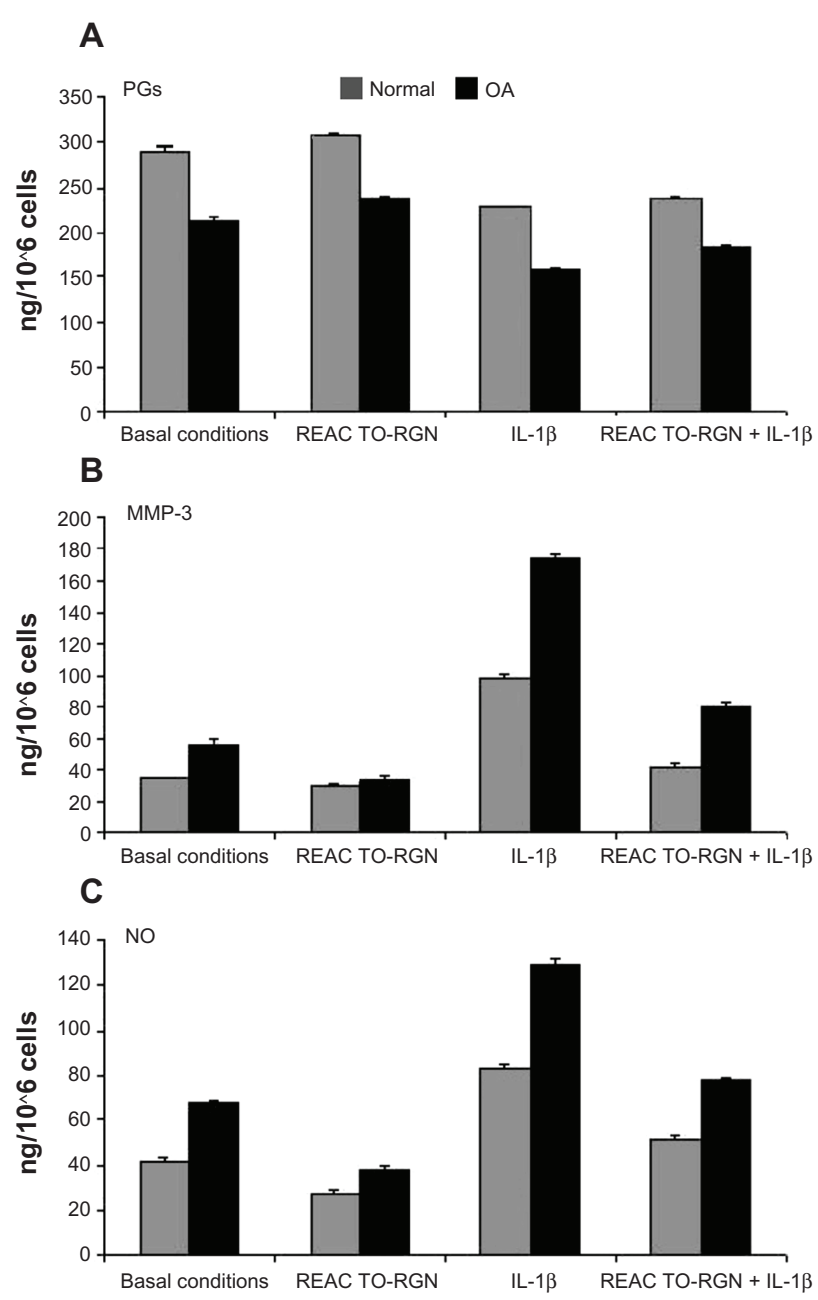

Figure I Effects of incubation of human normal and OA chondrocytes, at basal conditions; with REAC TO-RGN treatment alone; with IL-I $\beta(5 \mathrm{ng} / \mathrm{mL})$ alone; or coincubation with IL-I $\beta$ and REAC TO-RGN treatment for $48 \mathrm{~h}$ in the culture medium. (A) The quantity of PG (ng// $\left.10^{6}\right)$; (B) the MMP-3 level ( $\mathrm{ng} / 10^{6}$ cells); and (C) the NO production ( $\mathrm{ng} / 10^{6}$ cells).

Note: Data are expressed as mean values \pm SD.

Abbreviations: OA, osteoarthritis; REAC, radioelectric asymmetric conveyer technology; TO-RGN, Regenerative Tissue Optimization; IL, interleukin; PG, proteoglycan; MMP, metalloproteinase; NO, nitric oxide. 
both in the absence or presence of IL-1 $\beta$ (Figure 1B and C). Interestingly, the effect of REAC TO-RGN treatment on the decrease in MMP-3 levels was significantly higher in OA chondrocytes, as compared with normal cells, even after the addition of IL-1 $\beta(P<0.01)$. The level of NO in the OA chondrocyte culture significantly decreased with respect to that of normal chondrocytes after REAC TO-RGN at basal conditions $(P<0.01)$.

\section{TEM evaluation}

Normal chondrocytes showed a euchromatic nucleus and a cytoplasm containing abundant rough endoplasmic reticulum, Golgi bodies, and mitochondria (Figure not shown) (Table 1). Cultured OA chondrocytes showed a reduction in cytoplasmic components, such as Golgi bodies and mitochondria, and the presence of vacuoles (Figure 2A and Table 1). The REAC TO-RGN treatment, applied at basal conditions, improved the morphological status of the cultured normal and OA chondrocytes; in particular, in the OA cells (Figure 2B), a significantly increased number of mitochondria $(P<0.05)$ and Golgi bodies $(P<0.05)$ was displayed (Table 1).

In the normal and OA chondrocytes incubated with IL-1 $\beta$, a peculiar presence of vacuolization was observed (Figure 2C and Table 1); for this reason, the number of chondrocytes with an evident vacuolization (more than five vacuoles) was evaluated in each group; this number was significantly increased $(P<0.05)$ in both normal and OA chondrocytes incubated with IL-1 $\beta$ as compared with untreated cells. Moreover, the effect of IL-1 $\beta$ treatment on the number of chondrocytes with vacuolization was significantly higher in OA chondrocytes as compared to normal cells $(P<0.01)$. In the presence of IL- $1 \beta$ REAC, TO-RGN stimulus partially restored the cytoplasmic ultrastructure (Figure 2D) in both normal and OA chondrocytes, as shown by the decreased number of chondrocytes with vacuolization observed in both groups of cells. Moreover, in OA chondrocytes cultured in the presence of IL-1 $\beta$, the number of mitochondria and Golgi bodies was significantly increased $(P<0.05)$ after REAC TO-RGN treatment.

On the whole, significant differences emerged from the comparative analysis of the effects of REAC TO-RGN therapy on the number of mitochondria, Golgi bodies, and the number of vacuolized cells between normal and OA chondrocytes, confirming a higher receptivity of OA chondrocytes to REAC TO-RGN, both in basal conditions and in the presence of IL-1 $\beta(P<0.05$, mitochondria at basal conditions; $P<0.01$, mitochondria in the presence of IL-1 $\beta$ ), as compared with normal ones.

\section{Discussion}

Previous work has already demonstrated the effectiveness of a pulsed electromagnetic field (PEMF) to counteract IL-1 $\beta$ activity $^{19-21}$ and to regulate NO production, ${ }^{22}$ and also its effect on gene expression in human mesenchymal stem cells and chondrocytes. ${ }^{23}$

The present study shows that in both normal and OA chondrocytes, REAC TO-RGN exposure determined a reduction in the catabolic effect of IL-1 $\beta$, downregulating MMP-3 and NO production and increasing PG synthesis. We also observed that, in OA chondrocytes, the REAC TORGN-induced PG synthesis was higher as compared with normal cells. This effect may play an important role in the management of OA. It is well known that OA is an inflammatory disease that presents clinical features amenable to the limited regenerative capability of cartilage tissue and which is characterized by several structural changes in the cartilage tissue, including the degradation of cartilage matrix (due to the inability of OA chondrocytes to synthesize sufficient matrix to repair damaged tissues). ${ }^{24-26}$ Considering the adverse effect of anti-inflammatory drugs commonly used for OA handling, REAC treatments, having no side effect, can play an important role in the management of a disease that affects an increasing number of patients.

Table I Number (n) of mitochondria, Golgi bodies and presence of vacuolization (percentage, \%) in normal and OA chondrocytes, under basal conditions and after treatment with the REAC TO-RGN, in absence or presence of IL-I $\beta(5 \mathrm{ng} / \mathrm{mL})$

\begin{tabular}{|c|c|c|c|c|c|}
\hline & Group & Basal conditions & REAC TO-RGN & IL-I $\beta$ & REAC TO-RGN + IL-I $\beta$ \\
\hline \multirow[t]{2}{*}{ Mitochondria (n) } & Normal & 7.01 (0.43) & 7.99 (0.79) & $5.47(0.5 \mathrm{I})$ & $6.67(0.38)$ \\
\hline & $\mathrm{OA}$ & $3.27(0.37)$ & $5.43(0.40)^{*, \dagger}$ & $1.65(0.25)$ & $4.17(0.34)^{* *, \ddagger}$ \\
\hline \multirow[t]{2}{*}{ Golgi bodies (n) } & Normal & $3.52(0.44)$ & $3.80(0.62)$ & $2.77(0.54)$ & $3.23(0.60)$ \\
\hline & $\mathrm{OA}$ & $\mathrm{I} .53(0.37)$ & $3.17(0.57)^{*, \neq}$ & $0.77(0.33)$ & $2.43(0.33)^{* *, \ddagger}$ \\
\hline \multirow[t]{2}{*}{ Vacuolization $(>5)(\%)$} & Normal & $5.00(1.14)$ & $3.17(0.98)$ & $9.00(0.90)^{*}$ & $7.00(0.63)$ \\
\hline & $\mathrm{OA}$ & $12.00(1.10)$ & $7.00(0.90)^{\ddagger}$ & $29.67(1.37)^{*}$ & I $4.5(1.22)^{\ddagger}$ \\
\hline
\end{tabular}

Notes: The figures in brackets are the standard deviation values. For each group: $* P<0.05$, comparison vs basal conditions; $* * P<0.05$, comparison between REAC TORGN vs REAC TO-RGN + IL-I $\beta$. Considering the differences between normal and OA: ${ }^{\dagger} P<0.05 ; \neq P<0.01$.

Abbreviations: OA, Osteoarthritis; REAC, radio electric asymmetric conveyer technology; TO-RGN, Regenerative Tissue Optimization; IL, interleukin. 

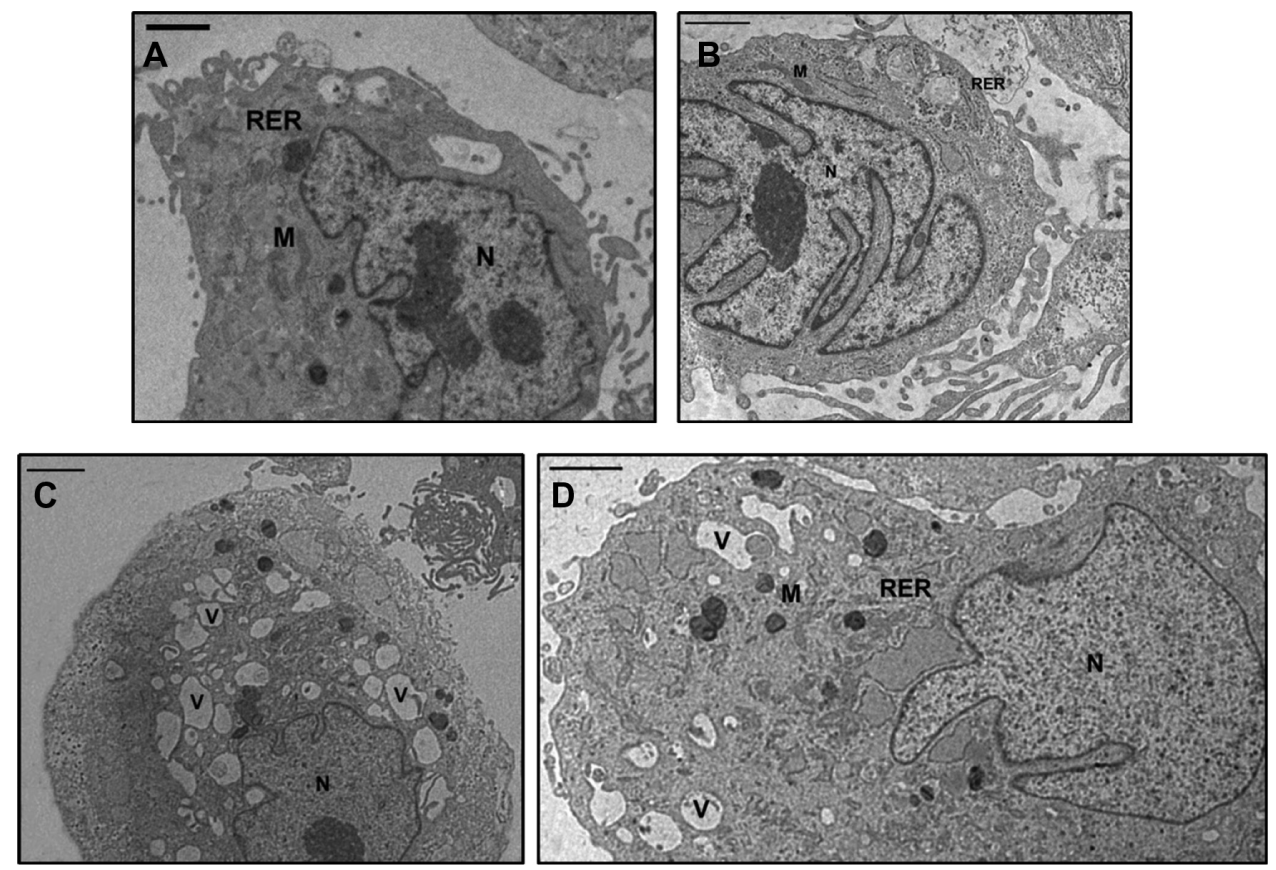

Figure 2 TEM micrographs of human OA chondrocytes. (A) Basal conditions: The cell shows an euchromatic nucleus (N), a reduction in cytoplasmic components, such as rough endoplasmic reticulum (RER) and mitochondria (M). The plasma membrane presents cytoplasmic processes. (B) Incubation with REAC TO-RNG: The nucleus $(\mathrm{N})$ is euchromatic; the cytoplasm shows a significant increase of the presence of rough endoplasmic reticulum and mitochondria (M). (C) Incubation with IL-I $\beta$ : The cytoplasm shows a diffuse vacuolization $(\mathrm{V})$ and it contains a reduced quantity of typical organelles, such as Golgi bodies, rough endoplasmic reticulum and mitochondria ( $M$ ). (D) Incubation with REAC TO-RNG + IL-I $\beta$ : The cell partially restores its morphology. The nucleus (N) is euchromatic, the cytoplasm shows a restored organization: a much reduced number of vacuoles $(\mathrm{V})$ is present, rough endoplasmic reticulum is abundant and mitochondria (M) are well shaped. The plasma membrane presents many cytoplasmic processes. Bar $=1 \mu \mathrm{m}$.

Abbreviations: TEM, transmission electron microscopy; OA, osteoarthritis; REAC, radioelectric asymmetric conveyer technology; TO-RGN, Regenerative Tissue Optimization; IL, interleukin; RER, rough endoplasmic reticulum; M, mitochondria; N, nucleus; V, vacuoles.

In a previous work, our group demonstrated a positive role of a physical approach, the pulsed signed therapy, in counteracting the matrix degeneration occurring in IL-1 $\beta$ OA chondrocytes in vitro. ${ }^{27}$ Moreover, studies by different authors have shown that under in vivo conditions, PEMF therapy could reduce pain and improve functional performance in patients with $\mathrm{OA},{ }^{28}$ and that under in vitro conditions, PEMF therapy may exert chondroprotective effects on human articular cartilage, particularly in the early stages of OA. ${ }^{29}$ However, PEMF treatments are different from REAC treatments for various reasons. First of all, PEMF therapy needs much longer-term exposure to treatment; secondly, at present there are no standardized treatment protocols (with specified characteristics of the applied PEMF) that have been tested in pilot studies and supported by compelling evidence. Finally, some clinical trials that have described positive results in $\mathrm{OA}$ applied electromagnetic fields over the levels recommended by the International Commission on Non-Ionizing Radiation Protection (http://www.who.int/peh-emf/about/WhatisEMF/ en/index4.html), ${ }^{30,31}$ an approach that may result in adverse effects on human health. In the present work, we analyzed the effect of REAC TO-RGN on the biochemical and morphological changes occurring in cultures of normal and OA chondrocytes, mimicking also the in vivo inflammatory milieu by the addition of IL-1 $\beta$. In fact, the proinflammatory cytokine IL-1 $\beta$ has chemical characteristics that influence protein catabolism and seems to be involved in the initiation and progression of OA, and also in NO production. ${ }^{32}$ Furthermore, increasing evidence suggests that chondrocyte death may contribute to the progression of OA. In particular, Blanco et $\mathrm{al}^{3}$ described apoptotic processes induced by NO in OA chondrocytes, which contribute to the decrease in the number of these extracellular matrix-producing cells. ${ }^{33}$ Within this context, in this study, we showed a decrease in NO production after REAC TO-RGN stimulation, both in IL-1 $\beta$-treated and IL-1 $\beta$-untreated OA and normal chondrocytes. Interestingly, the effect of REAC TO-RGN was significantly more pronounced in the OA chondrocytes than in normal cells. Furthermore, morphometric analysis of the normal and OA chondrocytes treated with IL- $1 \beta$ showed a statistically significant reduction in the number of mitochondria and Golgi bodies. A significant improvement in morphometric analysis and in the number of mithocondria and Golgi bodies was highlighted in cells treated with REAC TO-RGN treatment 
and with IL-1 $\beta$. The number of normal and OA chondrocytes with a diffuse vacuolization was also significantly reduced in the presence of REAC TO-RGN stimulus, determining a recovery of cellular features and confirming a shift toward anabolic activity. At the moment, we have not identified the exact sequence of events activated by REAC TO-RGN in chondrocytes. Although future investigations are needed in order to understand the molecular mechanisms involved in the positive effect observed in REAC TO-RGN-exposed chondrocytes, the present work clearly discloses the possibility that this instrument may represent a viable alternative to traditional OA therapy.

\section{Author contributions}

Salvatore Rinaldi and Vania Fontani developed the experimental design and contributed to the manuscript. Giulia Collodel and Nicola Antonio Pascarelli, conceived and executed most of the experimental plan, performed the transmission electron microscopy, and contributed to the manuscript. Antonella Fioravanti and Margherita Maioli conceived and executed most of the experimental plan and contributed to the manuscript. Antonello Lamboglia, Elena Moretti, Francesca Iacoponi, Sara Santaniello, Gianfranco Pigliaru, and Alessandro Castagna performed the experiments. Carlo Ventura designed/ supervised the project, and contributed to the manuscript.

\section{Disclosure}

Salvatore Rinaldi and Vania Fontani are the inventors of the radioelectric asymmetric conveyer. The authors report no other conflicts of interest in this work.

\section{References}

1. Srikanth VK, Fryer JL, Zhai G, Winzenberg TM, Hosmer D, Jones G. A meta-analysis of sex differences prevalence, incidence and severity of osteoarthritis. Osteoarthritis Cartilage. 2005;13(9):769-781.

2. Zhang Y, Jordan JM. Epidemiology of osteoarthritis. Clin Geriatr Med. 2010;26(3):355-369.

3. Blanco FJ, Ochs RL, Schwarz H, Lotz M. Chondrocyte apoptosis induced by nitric oxide. Am J Pathol. 1995;146(1):75-85.

4. Hashimoto H, Nagasawa T, Abe T, Shibata S, Mishima Y. Comparative studies of clinical findings and prognosis of polyarteritis nodosa, Wegener's granulomatosis, allergic granulomatous angiitis and malignant rheumatoid arthritis. Ryumachi. 1988;28(3):145-155. Japanese.

5. Goldring SR, Goldring MB. The role of cytokines in cartilage matrix degeneration in osteoarthritis. Clin Orthop Relat Res. 2004; (427 Suppl):S27-S36.

6. Abramson SB. Osteoarthritis and nitric oxide. Osteoarthritis Cartilage. 2008;16 Suppl 2:S15-S20.

7. Jordan KM, Arden NK, Doherty M, et al; Standing Committee for International Clinical Studies Including Therapeutic Trials ESCISIT. EULAR Recommendations 2003: an evidence based approach to the management of knee osteoarthritis: Report of a Task Force of the Standing Committee for International Clinical Studies Including Therapeutic Trials (ESCISIT). Ann Rheum Dis. 2003;62(12):1145-1155.
8. Savarino L, Fioravanti A, Leo G, Aloisi R, Mian M. Anthraquinone2,6-disulfonic acid as a disease-modifying osteoarthritis drug: an in vitro and in vivo study. Clin Orthop Relat Res. 2007;461:231-237.

9. Rinaldi S, Fontani V, inventors; Rinaldi S, Fontani V, assignees. Radioelectric asymmetric conveyer for therapeutic use. EP1301241 (B1). October 11, 2006.

10. Rinaldi S, Fontani V, inventors; Rinaldi S, Fontani V, assignees. Radioelectric asymmetric conveyer for therapeutic use. United States patent US 7333859. February 19, 2008.

11. Maioli M, Rinaldi S, Santaniello S, et al. Radiofrequency energy loop primes cardiac, neuronal, and skeletal muscle differentiation in mouse embryonic stem cells: a new tool for improving tissue regeneration. Cell Transplant. 2012;21(6):1225-1235.

12. Maioli M, Rinaldi S, Santaniello S, et al. Radio electric conveyed fields directly reprogram human dermal-skin fibroblasts towards cardiac-, neuronal-, and skeletal muscle-like lineages. Cell Transplant. Epub October 2, 2012.

13. Heinegard D, Saxne T. Connective tissue macromolecules as markers for tissue processes in joint disease. Eur J Rheumatol Inflamm. 1991; 11:91-99.

14. Altman R, Alarcón G, Appelrouth D, et al. The American College of Rheumatology criteria for the classification and reporting of osteoarthritis of the hip. Arthritis Rheum. 1991;34(5):505-514.

15. Lazzerini PE, Capecchi PL, Nerucci F, et al. Simvastatin reduces MMP-3 level in interleukin 1beta stimulated human chondrocyte culture. Ann Rheum Dis. 2004;63(7):867-869.

16. Ghasemi A, Zahediasl S. Preanalytical and analytical considerations for measuring nitric oxide metabolites in serum or plasma using the Griess method. Clin Lab. 2012;58(7-8):615-624.

17. Annefeld M. A new test method for the standardized evaluation of changes in the ultrastructure of chondrocytes. Int J Tissue React. 1985;7(4):273-289.

18. Fioravanti A, Collodel G, Petraglia A, Nerucci F, Moretti E, Galeazzi M. Effect of hydrostatic pressure of various magnitudes on osteoarthritic chondrocytes exposed to IL-1beta. Indian J Med Res. 2010;132:209-217.

19. Ongaro A, Pellati A, Setti S, et al. Electromagnetic fields counteract IL-1 $\beta$ activity during chondrogenesis of bovine mesenchymal stem cells. J Tissue Eng Regen Med. Epub December 17, 2012.

20. Rohde C, Chiang A, Adipoju O, Casper D, Pilla AA. Effects of pulsed electromagnetic fields on interleukin-1 beta and postoperative pain: a double-blind, placebo-controlled, pilot study in breast reduction patients. Plast Reconstr Surg. 2010;125(6):1620-1629.

21. Hammond DC. Discussion. Effects of pulsed electromagnetic fields on interleukin-1 beta and postoperative pain: a double-blind, placebocontrolled, pilot study in breast reduction patients. Plast Reconstr Surg. 2010;125(6):1630-1631.

22. Fitzsimmons RJ, Gordon SL, Kronberg J, Ganey T, Pilla AA. A pulsing electric field (PEF) increases human chondrocyte proliferation through a transduction pathway involving nitric oxide signaling. J Orthop Res. 2008;26(6):854-859.

23. Walther M, Mayer F, Kafka W, Schütze N. Effects of weak, low-frequency pulsed electromagnetic fields (BEMER type) on gene expression of human mesenchymal stem cells and chondrocytes: an in vitro study. Electromagn Biol Med. 2007;26(3):179-190.

24. Buckwalter JA, Mankin HJ. Articular cartilage: degeneration and osteoarthritis, repair, regeneration, and transplantation. Instr Course Lect. 1998;47:487-504.

25. Howell DS, Altman RD. Cartilage repair and conservation in osteoarthritis. A brief review of some experimental approaches to chondroprotection. Rheum Dis Clin North Am. 1993;19(3):713-724.

26. Moskowitz RW, Altman RD, Hochberg MC, Buckwalter JA, Goldberg VM. Osteoarthritis: Diagnosis and Medical/Surgical Management, 4th ed. Philadelphia: Lippincott Williams \& Wilkins; 2007.

27. Fioravanti A, Nerucci F, Collodel G, Markoll R, Marcolongo R. Biochemical and morphological study of human articular chondrocytes cultivated in the presence of pulsed signal therapy. Ann Rheum Dis. 2002;61(11):1032-1033. 
28. Ryang We S, Koog YH, Jeong KI, Wi H. Effects of pulsed electromagnetic field on knee osteoarthritis: a systematic review. Rheumatology (Oxford). Epub April 13, 2012.

29. Ongaro A, Pellati A, Masieri FF, et al. Chondroprotective effects of pulsed electromagnetic fields on human cartilage explants. Bioelectromagnetics. 2011;32(7):543-551.

30. Trock DH, Bollet AJ, Markoll R. The effect of pulsed electromagnetic fields in the treatment of osteoarthritis of the knee and cervical spine. Report of randomized, double blind, placebo controlled trials. J Rheumatol. 1994;21(10):1903-1911.

31. Thamsborg G, Florescu A, Oturai P, Fallentin E, Tritsaris K, Dissing S. Treatment of knee osteoarthritis with pulsed electromagnetic fields: a randomized, double-blind, placebo-controlled study. Osteoarthritis Cartilage. 2005;13(7):575-581.
32. Tetlow LC, Adlam DJ, Woolley DE. Matrix metalloproteinase and proinflammatory cytokine production by chondrocytes of human osteoarthritic cartilage: associations with degenerative changes. Arthritis Rheum. 2001;44(3):585-594.

33. Clancy R, Rediske J, Koehne C, et al. Activation of stress-activated protein kinase in osteoarthritic cartilage: evidence for nitric oxide dependence. Osteoarthritis Cartilage. 2001;9(4):294-299.

\section{Publish your work in this journal}

Clinical Interventions in Aging is an international, peer-reviewed journal focusing on evidence-based reports on the value or lack thereof of treatments intended to prevent or delay the onset of maladaptive correlates of aging in human beings. This journal is indexed on PubMed Central, MedLine, the American Chemical Society's 'Chemical Abstracts
Service' (CAS), Scopus and the Elsevier Bibliographic databases. The manuscript management system is completely online and includes a very quick and fair peer-review system, which is all easy to use. Visit http://www.dovepress.com/testimonials.php to read real quotes from published authors.

Submit your manuscript here: http://www.dovepress.com/clinical-interventions-in-aging-journal 